\title{
A regularised singularity approach to phoretic problems
}

\author{
Thomas D. Montenegro-Johnson ${ }^{1}$, Sébastien Michelin ${ }^{2}$, and Eric Lauga ${ }^{1}$ \\ 1 Department of Applied Mathematics and Theoretical Physics, University of Cambridge, CMS, Wilberforce Rd, Cambridge, \\ CB3 0WA, UK \\ 2 LadHyX - Département de Mécanique, Ecole polytechnique - CNRS, 91128 Palaiseau Cedex, France
}

November 10, 2015

\begin{abstract}
An efficient, accurate, and flexible numerical method is proposed for the solution of the swimming problem of one or more autophoretic particles in the purely-diffusive limit. The method relies on successive boundary element solutions of the Laplacian and the Stokes flow equations using regularised Green's functions for swift, simple implementations, an extension of the well-known method of "regularised stokeslets" for Stokes flow problems. The boundary element method is particularly suitable for phoretic problems, since no quantities in the domain bulk are required to compute the swimming velocity. For time-dependent problems, the method requires no re-meshing and simple boundaries such as a plane wall may be added at no increase to the size of the linear system through the method of images. The method is validated against two classical examples for which an analytical or semi-analytical solution is known, a two-sphere system and a Janus particle, and provides a rigorous computational pipeline to address further problems with complex geometry and multiple bodies.
\end{abstract}

\section{Introduction}

\subsection{Background}

Artificial self-propelled synthetic devices, or swimmers, at microscopic scales have recently received increasing study, motivated both by advances in the understanding of biological locomotion [1] and potential engineering or biomedical applications [2]. Such synthetic systems can be roughly divided into two main categories, depending on whether they are externally actuated (e.g. by a rotating magnetic field [3,4]) or fuel-based [5].

The principal advantage of fuel-based swimmers is that they utilise properties of the immediate environment, rather than an externally-applied driving mechanism, in order to generate propulsion. An important class of fuelbased swimmers are those that exploit phoretic mechanisms, e.g. Janus particles [6]. The ability of phoretic swimmers to self-propel depends on two chemical properties of their surface: mobility and activity. Interaction with the solute species in the fluid within a thin interaction layer leads to local pressure imbalances. A net slip flow (and locomotion) develops at their boundary due to local solute concentration gradients (diffusiophoresis), temperature gradients (thermophoresis) or electric field (electrophoresis) [7]; a property of the surface generically termed as mobility. In order to create the required gradients, the particle must also be active, i.e. modify the local field gradients through chemical reaction catalysed at its surface or heat emission. Canonical experimental phoretic systems include bi-metallic $\mathrm{Au}-\mathrm{Pt}$ rods or spheres [8] and PS-Pt coated spheres [9]. Whilst the precise details of the physico-chemical mechanisms underlying these systems are still under debate [10], they have garnered much interest, particularly as a means to study collective dynamics at the micron-scale [11].

The recently-proposed continuum framework for selfdiffusiophoretic particles [12] relies on three assumptions: (i) the solute particle interaction layer is infinitely thin, and thus phoretic effects are incorporated through a surface slip velocity; (ii) particle length scales are microscopic, so that the Péclet number is zero and (iii) the catalytic portion of the surface absorbs or releases chemical at a fixed rate. Within this framework, obtaining the particle swimming velocity requires solution of the diffusive solute dynamics, and the boundary-driven Stokes flow problem in the fluid domain. Hitherto, detailed modelling of phoretic particles has largely been analytical, focusing on simple geometries [12-14]. Numerical studies have typically used non-continuum methods for individual particles [15], or considered collective or non-trivial confinement after much simplification of the solute-fluid dynamics [16-18]. In contrast, boundary element techniques, which solve the solute-fluid dynamics directly, are only beginning to be utilised [19-21].

In this paper, we develop a versatile numerical framework based on boundary integral methods and a regularised solution to the Laplace equation, inspired by classical work on regularised solutions to Stokes flow $[22,23]$. After a presentation of the approach and numerical techniques, the method is validated against analytical solutions for a two-sphere system and a classic Janus particle. 


\subsection{Problem formulation}

Following previous studies [14,21], we consider the purely diffusive limit of the self-diffusiophoretic locomotion problem: a rigid particle of typical size $L$ achieves selfpropulsion through its interaction with a diffusing solute species present in the fluid. Neglecting solute advection, the solute concentration completely decouples from the flow dynamics and satisfies the Laplace equation,

$$
D \nabla^{2} c=0 .
$$

The phoretic particle's chemical properties are characterized by the activity $\mathcal{A}(\mathbf{x})$ and mobility $\mathcal{M}(\mathbf{x})$ of its surface $S$, which may be homogeneous [14] or spatially-dependent, as for Janus particles [6]. Hence, at the particle's surface, solute is released or absorbed with flux,

$$
D \mathbf{n} \cdot \nabla c=-\mathcal{A}(\mathbf{x}) .
$$

Local gradients of solute concentration at the surface result in imbalances in the pressure field that drive a flow within the interaction layer, where the solute-particle interactions dominate. In the thin-interaction-layer limit, this is equivalent to a net phoretic slip velocity [7]

$$
\mathbf{u}=\mathcal{M}(\mathbf{x})(\mathbf{I}-\mathbf{n n}) \cdot \boldsymbol{\nabla} c \text { on } S,
$$

that in turn drives a fluid flow around the particle. Because of the micrometric size of such particles, the flow dynamics is governed by Stokes flow equations

$$
\eta \nabla^{2} \mathbf{u}=\nabla p, \quad \boldsymbol{\nabla} \cdot \mathbf{u}=0
$$

for which fluid and solid inertia are negligible when compared with viscous stresses. For freely-swimming particles, the total hydrodynamic force and torque must vanish, which closes the system of equations for the translational and angular velocities $(\mathbf{U}, \boldsymbol{\Omega})$ of the particle. In the following, the problem is non-dimensionalised using $L$, $\mathcal{A} L / D$ and $\mathcal{A} \mathcal{M} / D$ as characteristic length, concentration and velocity scales, respectively.

\section{Method}

A popular numerical means of solving the Laplace and Stokes flow equations is the boundary element method, which effectively replaces the partial differential equations (1) and (4) over a three-dimensional domain by integral equations over the particle's boundary. This reduction makes the boundary element method particularly suitable for phoretic problems, which are completely characterized by boundary values of the different fields and do not require an explicit description of the bulk distributions [21]; indeed the coupling between the solute and Stokes flow problems, eq. (3), only requires the value of the concentration along the boundary. Furthermore, since the three-dimensional domain is not meshed, no re-meshing is required when analysing the trajectories of several rigid particles.

\subsection{Finding the concentration: regularised sourcelets}

We propose a boundary element method to solve Laplace's equation inspired by the regularised stokeslet method for Stokes flow. To this end, regularised free space Green's functions are defined that satisfy

$$
\begin{aligned}
\nabla^{2} G^{\epsilon}\left(\mathbf{x}-\mathbf{x}_{0}\right) & =-\phi_{\epsilon}\left(\mathbf{x}-\mathbf{x}_{0}\right) \\
\nabla^{2} \mathbf{K}^{\epsilon}\left(\mathbf{x}-\mathbf{x}_{0}\right) & =-\nabla \phi_{\epsilon}\left(\mathbf{x}-\mathbf{x}_{0}\right)
\end{aligned}
$$

where $\phi_{\epsilon}$ is a 'blob' source located at $\mathbf{x}_{0}$, with small regularisation parameter $\epsilon$. In the standard boundary element method, the use of singular Green's functions necessitates careful treatment of surface integrals. This regularisation allows the use of simple, standard quadrature routines for all surface integrals in the regularised boundary integral equation [24]. As is common for the method of regularised stokeslets [23], we choose a regularisation function of the form

$$
\phi_{\epsilon}\left(\mathbf{x}-\mathbf{x}_{0}\right)=\frac{15 \epsilon^{4}}{8 \pi r_{\epsilon}^{7}}, \quad r_{\epsilon}^{2}=r^{2}+\epsilon^{2},
$$

where $r=\left|\mathbf{x}-\mathbf{x}_{0}\right|$. For this choice of regularisation function, the solutions to eqs (5) are

$$
\begin{aligned}
G^{\epsilon}\left(\mathbf{x}-\mathbf{x}_{0}\right) & =-\frac{2 r^{2}+3 \epsilon^{2}}{8 \pi r_{\epsilon}^{3}}, \\
K_{j}^{\epsilon}\left(\mathbf{x}-\mathbf{x}_{0}\right) & =r_{j} \frac{2 r^{2}+5 \epsilon^{2}}{8 \pi r_{\epsilon}^{5}}
\end{aligned}
$$

with $r_{j}=\left(\mathbf{x}-\mathbf{x}_{0}\right)_{j}$. Note that for $r \neq 0$, as $\epsilon \rightarrow 0$ we recover the well-known fundamental solutions of a point sink and source dipole, respectively,

$$
\begin{aligned}
& \lim _{\epsilon \rightarrow 0} G^{\epsilon}\left(\mathbf{x}, \mathbf{x}_{0}\right)=G^{0}\left(\mathbf{x}, \mathbf{x}_{0}\right)=-\frac{1}{4 \pi r}, \\
& \lim _{\epsilon \rightarrow 0} K_{j}^{\epsilon}\left(\mathbf{x}, \mathbf{x}_{0}\right)=K_{j}^{0}\left(\mathbf{x}, \mathbf{x}_{0}\right)=\frac{r_{j}}{4 \pi r^{3}} .
\end{aligned}
$$

Following the method of regularised stokeslets, these regularised Green's functions are used to construct a regularised boundary integral equation for the diffusion equation,

$$
\begin{aligned}
\int_{V} c(\mathbf{x}) \phi_{\epsilon}\left(\mathbf{x}-\mathbf{x}_{0}\right) \mathrm{d} V_{x}=\int_{S} & {\left[c(\mathbf{x}) \mathbf{K}^{\epsilon}\left(\mathbf{x}, \mathbf{x}_{0}\right) \cdot \mathbf{n}(\mathbf{x})\right.} \\
& \left.-\frac{\partial c(\mathbf{x})}{\partial \mathbf{n}} G^{\epsilon}\left(\mathbf{x}, \mathbf{x}_{0}\right)\right] \mathrm{d} S_{x},
\end{aligned}
$$

where $\mathbf{n}$ is the normal to the swimmer surface pointing into the fluid, and $\mathbf{x}_{0}$ is a fixed point. In the singular boundary integral method, the left-hand side term is computed exactly

$$
\int_{V} c(\mathbf{x}) \delta\left(\mathbf{x}-\mathbf{x}_{0}\right) \mathrm{d} V_{x}=\lambda c\left(\mathbf{x}_{0}\right),
$$

where $\lambda=0,1 / 2,1$ depending on whether the evaluation point $\mathbf{x}_{0}$ is inside, on, or outside the boundary, respectively. In the regularised method, this critical step is less 
straightforward. Firstly, assuming slow variations of the concentration field at the scale of $\epsilon$, we obtain

$$
\int c(\mathbf{x}) \phi_{\epsilon}\left(\mathbf{x}-\mathbf{x}_{0}\right) \mathrm{d} V_{x} \approx c\left(\mathbf{x}_{0}\right) \int \phi_{\epsilon}\left(\mathbf{x}-\mathbf{x}_{0}\right) \mathrm{d} V_{x},
$$

which is similar to the singular case for flat surfaces. Then, the integral on the right-hand side must be evaluated. When the mean curvature of the surface is negligible in comparison to $\epsilon^{-1}$, the approximation $\lambda=0$ (inside), $1 / 2$ (on the surface) and 1 (outside) provides sufficient accuracy. Note that here, inside/outside (resp. on the surface) is understood as much further (resp. closer) from the surface than $\epsilon$. For a smooth surface, the accuracy can be improved by taking into account the curvature of the particle's surface through a Taylor's series expansion of the surface's geometry. At leading order, the value of $\lambda$ for an evaluation point on the boundary becomes

$$
\lambda \approx \frac{1}{2}+\frac{\kappa \epsilon}{4}
$$

where $\kappa$ is the mean local curvature of the surface, counted positively when the particle is locally convex. For a sphere with $\kappa \epsilon=0.01$, the integral of $\phi_{\epsilon}\left(\mathbf{x}-\mathbf{x}_{0}\right)$ can be computed exactly, and the above expansion is accurate to $\mathcal{O}\left(10^{-8}\right) \%$. However, this approximation clearly breaks down near a cusp or a corner, where additional care should be taken.

It is a relatively simple matter to adjust the boundary integral formulation to include simple confining boundaries. For instance, the presence of a plane wall or freesurface may be modelled by replacing the Green's functions (7) in eq. (9) with the appropriate half-plane solutions. Crucially, this substitution results in no increase to the size of the resultant linear system, and multi-particle systems over a boundary may be modelled as readily as in free space. Such images are commonly employed for point singularities in electrostatics, and also for studying viscous swimmers and cilium-induced flow [25]. However, in contrast with regularised image systems for Stokes flows [26], the half-space Green's functions for the Laplacian above a plane boundary located at $z=0$ are remarkably simple,

$$
\begin{aligned}
& G^{\epsilon_{w}}\left(\mathbf{x}-\mathbf{x}_{0}\right)=G^{\epsilon}\left(\mathbf{x}-\mathbf{x}_{0}\right)+\beta G^{\epsilon}\left(\mathbf{x}-\mathbf{x}_{\mathrm{im}}\right), \\
& K_{j}^{\epsilon_{w}}\left(\mathbf{x}-\mathbf{x}_{0}\right)=K_{j}^{\epsilon}\left(\mathbf{x}-\mathbf{x}_{0}\right)+\beta \gamma_{j} K_{j}^{\epsilon}\left(\mathbf{x}-\mathbf{x}_{\mathrm{im}}\right),
\end{aligned}
$$

where $\mathbf{x}_{\mathrm{im}}=\left(x_{0}, y_{0},-z_{0}\right)$ and $\gamma_{1}=\gamma_{2}=1, \gamma_{3}=-1$. The constant $\beta$ takes the value $\beta=-1$ for absorbing boundaries, $c(x, y, 0)=0$, and $\beta=+1$ for no flux boundaries $\partial c / \partial z=0$.

\subsection{Calculating the slip velocity}

Once the surface concentration has been obtained, its gradient along the surface can be calculated through evaluation of the integral,

$$
\begin{array}{r}
\lambda \nabla c\left(\mathbf{x}_{0}\right)=\int_{S} c(\mathbf{x}) \mathbf{L}^{\epsilon}\left(\mathbf{x}, \mathbf{x}_{0}\right) \cdot \mathbf{n}(\mathbf{x}) \\
-\frac{\partial c(\mathbf{x})}{\partial \mathbf{n}} \mathbf{K}^{\epsilon}\left(\mathbf{x}, \mathbf{x}_{0}\right) \mathrm{d} S_{x},
\end{array}
$$

which can be formally obtained by differentiating eq. (9) with respect to $\mathbf{x}_{0}$. The concentration gradient $\boldsymbol{\nabla} c(\mathbf{x})$ is then substituted into eq. (3) to give the surface slip velocity. Here, we have neglected an additional left hand side term $c\left(\mathbf{x}_{0}\right) \nabla \lambda\left(\mathbf{x}_{0}\right)$ : we are only interested in the part of these gradients tangential to the surface, and along smooth surfaces $\lambda$ changes very slowly. In free space, the Green's function $\mathbf{L}^{\epsilon}\left(\mathbf{x}, \mathbf{x}_{0}\right)$ is the derivative of the dipole

$$
\begin{aligned}
\frac{\partial K_{i}^{\epsilon}}{\partial x_{j}}=L_{i j}^{\epsilon} & =\delta_{i j} \frac{2 r^{2}+5 \epsilon^{2}}{8 \pi r_{\epsilon}^{5}} \\
& +r_{i} r_{j}\left(\frac{1}{2 \pi r_{\epsilon}^{5}}-\frac{10 r^{2}+25 \epsilon^{2}}{8 \pi r_{\epsilon}^{7}}\right)
\end{aligned}
$$

where $\delta_{i j}$ is the Kronecker delta tensor. Once eq. (9) has been solved to obtain the concentration field, $c(\mathbf{x})$ and $\partial c(\mathbf{x}) / \partial \mathbf{n}$ are known on the particle's boundary. Therefore, computing the concentration gradient (14) does not require solving another integral equation, or inverting a linear system. The singularity is an order higher than in the concentration calculation, however the regularisation ensures that the Green's functions remain integrable; either higher-order quadrature or increased regularisation is sufficient for convergence. If the value of the regularisation parameter $\epsilon$ is increased, $\lambda$ too must be recalculated for the gradient evaluation only. We have employed this increased regularisation in the results that follow. For flat surfaces, it is worth noting that an alternative simple approach to calculating the slip velocity can be obtained through finite differences of interpolated values of the surface concentration.

\subsection{Finding the surface traction: regularised stokeslets}

To solve for the particle's swimming velocity and the induced fluid flow, we employ the regularised stokeslet boundary element method [23,24]. This method has the advantage that finding the velocity of the particle only requires the tractions on the particle surface, and not the flow field in the bulk. Furthermore, much of the numerical code employed to solve the Laplace problem can be reused, with a substitution of the appropriate Green's functions.

For Stokes flow, eqs (4), the fluid velocity at any point in the domain can be evaluated through integrals of stokeslets $\mathbf{S}$ and stresslets $\mathbf{T}$ over the surface of the particles,

$$
\begin{aligned}
\lambda u_{j}\left(\mathbf{x}_{0}\right)=\int_{S} & S_{i j}^{\epsilon}\left(\mathbf{x}, \mathbf{x}_{0}\right) f_{i}(\mathbf{x}) \\
& -u_{i}(\mathbf{x}) T_{i j k}^{\epsilon}\left(\mathbf{x}, \mathbf{x}_{0}\right) n_{k}(\mathbf{x}) \mathrm{d} S_{x},
\end{aligned}
$$

where $\lambda, \mathbf{x}_{0}$ and $\mathbf{n}$ are as in eq. (9). We utilise the same regularisation as for the Laplacian (6), for which we have 
regularised stokeslet $S_{i j}^{\epsilon}$ and stresslet $T_{i j k}^{\epsilon}[23]$

$$
\begin{aligned}
S_{i j}^{\epsilon}\left(\mathbf{x}, \mathbf{x}_{0}\right)= & \frac{\delta_{i j}\left(r^{2}+2 \epsilon^{2}\right)+r_{i} r_{j}}{r_{\epsilon}^{3}}, \\
T_{i j k}^{\epsilon}\left(\mathbf{x}, \mathbf{x}_{0}\right)= & -\frac{6 r_{i} r_{j} r_{k}}{r_{\epsilon}^{5}} \\
& -\frac{3 \epsilon^{2}\left(r_{i} \delta_{j k}+r_{j} \delta_{i k}+r_{k} \delta_{i j}\right)}{r_{\epsilon}^{5}} .
\end{aligned}
$$

Substituting $\mathbf{u}(\mathbf{x})=\mathbf{u}^{s}(\mathbf{x})+\mathbf{U}+\boldsymbol{\Omega} \wedge\left(\mathbf{x}-\mathbf{x}_{\mathbf{c}}\right)$ for surface slip velocity $\mathbf{u}^{s}$, swimming translational and angular velocities $\mathbf{U}, \boldsymbol{\Omega}$, and particle centre $\mathbf{x}_{\mathbf{c}}$ into eq. (16), we see

$$
\begin{aligned}
& \lambda u_{j}^{s}\left(\mathbf{x}_{0}\right)+\int_{S} u_{i}^{s}(\mathbf{x}) T_{i j k}^{\epsilon}\left(\mathbf{x}, \mathbf{x}_{0}\right) n_{k}(\mathbf{x}) \mathrm{d} S_{x} \\
& =\int_{S} S_{i j}^{\epsilon}\left(\mathbf{x}, \mathbf{x}_{0}\right) f_{i}(\mathbf{x}) \\
& -\left[U_{i}+\left(\boldsymbol{\Omega} \wedge\left[\mathbf{x}_{0}-\mathbf{x}_{c}\right]\right)_{i}\right] T_{i j k}^{\epsilon}\left(\mathbf{x}, \mathbf{x}_{0}\right) n_{k}(\mathbf{x}) \mathrm{d} S_{x}
\end{aligned}
$$

where the surface tractions $\mathbf{f}$ and swimming velocities $\mathbf{U}, \boldsymbol{\Omega}$ are unknowns. The linear system is closed for the unknown swimming velocities by enforcing the zero net force and torque conditions

$$
\int_{S} \mathbf{f}(\mathbf{x}) \mathrm{d} S_{x}=\mathbf{0}, \quad \int_{S}\left(\mathbf{x}-\mathbf{x}_{c}\right) \wedge \mathbf{f}(\mathbf{x}) \mathrm{d} S_{x}=\mathbf{0},
$$

as constraints in the matrix system [27] that arises from the discretisation of eq. (18).

\subsection{Numerical implementation}

We now discuss the numerical implementation of this framework, which is freely available as the package "RegBEM Phoretic" from the Matlab file exchange [28].

The first step in solving the phoretic locomotion problem with the boundary element method is to define the computational mesh of the particle surfaces. This part of the procedure is essentially distinct from the boundary element solver, and many automatic mesh generation packages are available. We use the Matlab package DistMesh [29] to generate a flat triangular representation of our surfaces, and then custom routines are used to extend this to a piecewise quadratic surface description (fig. 1). A quadratic geometric representation of the surface allows the geometry to be better captured by fewer elements, and gives a significant increase in solution accuracy [30]. Integration of the Green's functions over each element is performed numerically using adaptive Fekete quadrature [31,32], with lookup table routines written by John Burkardt [33]. The coordinates of these quadrature points are projected from the canonical triangle to each quadratic element using the routines abc and interp_p from the BEMLIB library [34], adapted for use in Matlab.

Whilst the mesh is geometrically quadratic, the discretisation of the concentration $c(\mathbf{x})$ and the surface tractions $\mathbf{f}(\mathbf{x})$ can be defined independently, according to the
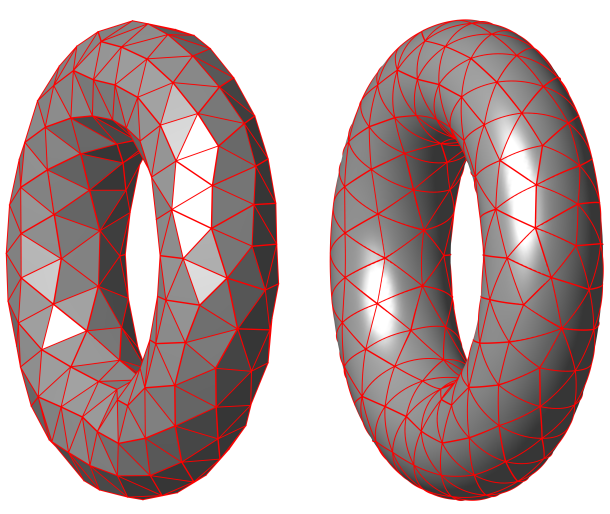

Fig. 1. The surface mesh description. The geometrically quadratic mesh (top right) provides a much more faithful representation of the particle surface than a linear mesh with the same number of elements (top left).

user's needs. A common implementation of the boundary element method is with constant panels; the unknown field on the surface is discretised as constant over each element, collocated at the element centroid [24,32]. The constant panel method has a simple implementation, and can provide a relatively high level of accuracy. However, phoretic problems involve solving a series of subproblems: finding an unknown surface concentration, evaluating the concentration gradient to give a slip velocity, and then finding the unknown surface tractions and propulsion velocity arising from this surface slip. Achieving high accuracy at each step is essential in order to avoid propagating errors. Thus we employ a linear panel boundary element method, where the surface unknowns are discretised as linear over each element.

The linear panel method confers a number of advantages over the constant panel method. Firstly, it provides a higher-order, more accurate representation of the unknown surface field. Secondly, each element has exactly three linear nodes where the unknowns are collocated, the vertices of the triangle, but for closed surfaces each vertex is shared between 6 elements, on average. Thus, for any given mesh the number of degrees of freedom for the linear panel method is approximately half that of the constant panel mesh, reducing the time to solve the linear system by approximately a factor of 8 . Finally, Fekete quadrature points are clustered near the element vertices, leading to more accurate evaluation of the nearly singular element integrals for any given number of quadrature points, when compared to the constant panel method.

The element-based discretisation of the concentration and surface tractions ensures that the solution space is decoupled from the numerical quadrature used to evaluate the surface integrals. Whilst more complicated than traditional implementations of regularised stokeslets [23, $35]$, this boundary element implementation has the advantage of maintaining accuracy whilst greatly reducing the size of the linear system to be solved [24]. Furthermore, it allows for adaptive quadrature to be used for integrals 


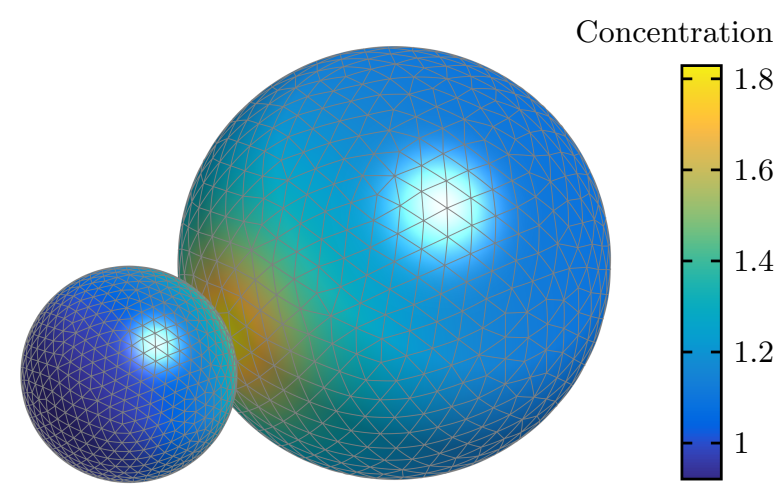

Fig. 2. Surface concentration of the two-sphere system with $R_{2}=0.5$ and $d_{c}=0.1$.

over elements far from the collocation point, significantly reducing the time required to assemble the matrix system. The simulations in the following section typically run in around a minute on a 16GB Macbook Retina Pro (2013), though if less accuracy is required simulation time may be of the order of seconds.

\section{Validation of numerical scheme}

We now proceed with a validation of our method against well-established analytical results for a two-sphere system [14], and a Janus particle [12]. The two sphere system has non-trivial geometry with simple surface chemistry resulting in constant flux. In contrast, the Janus particle has a simple geometry with discontinuous surface chemistry, resulting in zero-flux and unit flux hemispheres.

\subsection{A two-sphere system}

We first validate our method against the semi-analytical solution of the phoretic problem considered in Ref. [14]; a swimmer comprising two chemically-homogeneous spheres with radii $\left(R_{1}=1, R_{2}\right)$, rigidly linked and separated by a contact distance $d_{c}$ (Figure 2). The performance of the code is examined by comparing the surface concentration, slip velocity and swimming velocity with the analytical result. The following computations were performed with 1320 linear nodes per sphere, with regularisation $\epsilon=0.002 R_{1,2}$ for the concentration and traction calculations, and $\epsilon=0.01 R_{1,2}$ for the slip velocity evaluation.

The surface concentration and slip velocity as a function of the polar angle $\theta$ from the midline between the spheres is shown in figure (3). The solution is given for almost-touching spheres $d_{c}=0.1$ and for $d_{c}=R_{2}=0.5$, and compared to the analytical result. The mean relative errors for $d_{c}=0.1$ on the second sphere are $0.05 \%$ for the surface concentration and $0.2 \%$ for the slip velocity, while for $d_{c}=0.5$ the mean relative errors are $0.05 \%$ and $0.2 \%$, showing excellent agreement with analytical values.

The self-propulsion velocity of the two-sphere assembly as a function of contact distance $d_{c}$ is then computed,
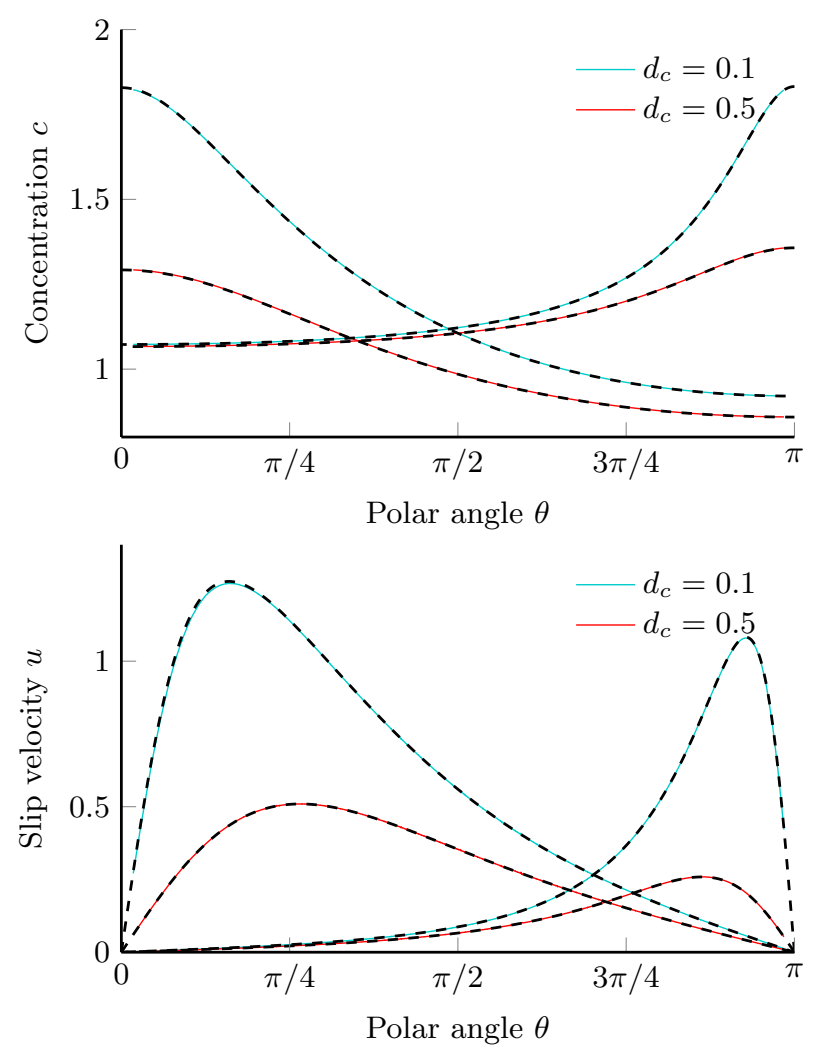

Fig. 3. Surface concentration (upper) and slip velocity (lower) of the two-sphere system, for $d_{c}=0.1$ (cyan) and $d_{c}=0.5$ (red). Sphere 1 takes its peak values on the right of the plot, and the smaller sphere 2 on the left. The analytical result [14] is virtually indistinguishable, and shown for reference in black.

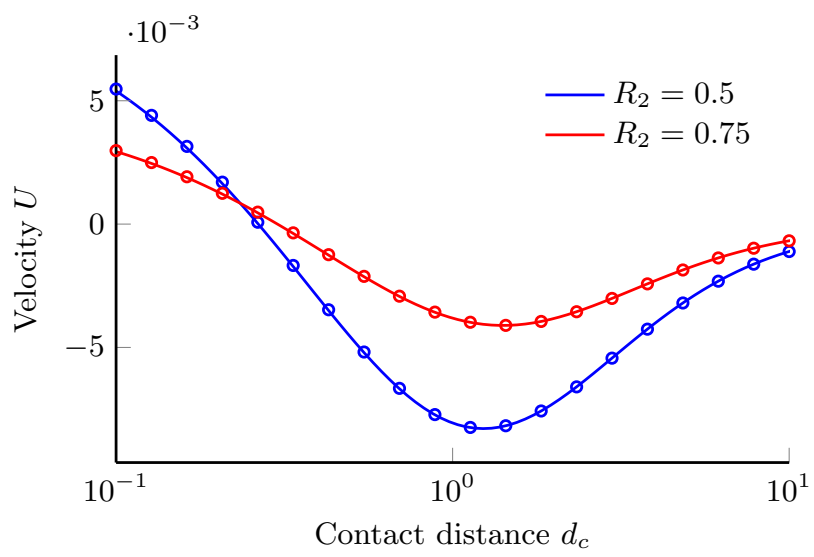

Fig. 4. Self-propulsion velocity of a two-sphere rigid system as function of the contact distance $d_{c}$, with $R_{1}=1, R_{2}=0.5$ (blue) and $R_{2}=0.75$ (red), obtained numerically using the present approach (crosses) and analytically [14] (solid lines).

for $R_{2}=0.5$ and $R_{2}=0.75$, and compared to the results of Michelin and Lauga [14]; the numerical results are in good agreement with the analytical prediction (Figure 4). Because the swimming velocity may vanish for certain distances, the relative error is evaluated here by dividing the absolute error by the average of the absolute speed in our sample, obtaining a maximum relative error of $1.5 \%$ at 

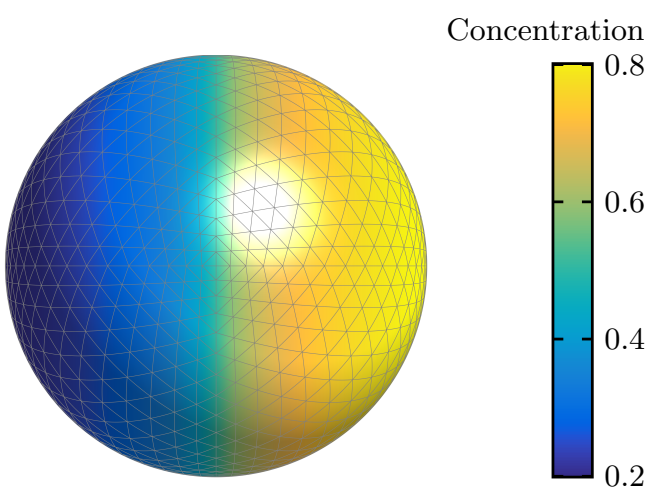

Fig. 5. Surface concentration of a Janus particle, calculated using our numerical method.

the closest contact distance of $d_{c}=0.1$, the system shown in figure 2. For distances of $\mathcal{O}(1)$ or greater, the relative error is less than $0.1 \%$.

\subsection{Validation: A spherical Janus particle}

A more common route to breaking symmetry and achieving self-propulsion in autophoretic system is chemical patterning: such Janus particles [6] have different chemical properties (i.e. activity and mobility) on their two halves, and in particular may catalyze a chemical reaction only on one of their hemispheres (fig. 5). In such cases, the normal flux of solute into the fluid domain is discontinuous at the edge of the coating, and it is important that our method is able to handle such discontinuities.

For an axisymmetric, spherical Janus particle with uniform mobility $\mathcal{M}=1$ and a hemispherical reactive cap with activity $\mathcal{A}=1$ (the other hemisphere being inert, $\mathcal{A}=0$ ), the surface concentration is given by $[12,36]$

$$
c(r, \mu)=\sum_{p=0}^{\infty} c_{p}(r) L_{p}(\mu), \quad c_{p}(r)=\frac{k_{p}}{(p+1) r^{p+1}},
$$

where $L_{p}(\mu)$ is the $p^{\text {th }}$ Legendre polynomial with $\mu=\cos \theta$ the cosine of the polar angle, and the coefficients $k_{p}$ are obtained as

$$
\begin{gathered}
k_{0}=\frac{1}{2}, \quad k_{2 q}=0 \\
k_{2 q-1}=(-1)^{q+1} \frac{4 q-1}{4 q-2} \frac{(2 q) !}{\left(2^{q} q !\right)^{2}}, \quad q \geq 1 .
\end{gathered}
$$

The slip velocity can be easily obtained by taking the gradient of eq. (20). For a homogenous mobility, the swimming velocity is obtained in terms of $c$ as [37]

$$
U=-\int_{-1}^{1} \mu c(1, \mu) \mathrm{d} \mu=\frac{k_{1}}{3}=\frac{1}{4} .
$$

The series solution for the concentration converges sufficiently quickly for a quantitative comparison, however

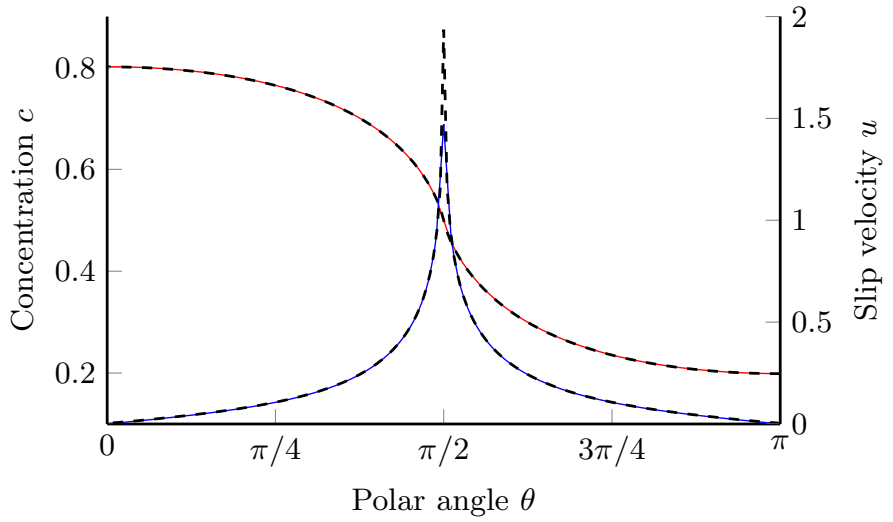

Fig. 6. Surface concentration (red) and slip velocity (blue) of a Janus particle with uniform mobility and a hemispherical cap with unit activity. Analytical values calculated from the series are overlaid with dashed black lines.

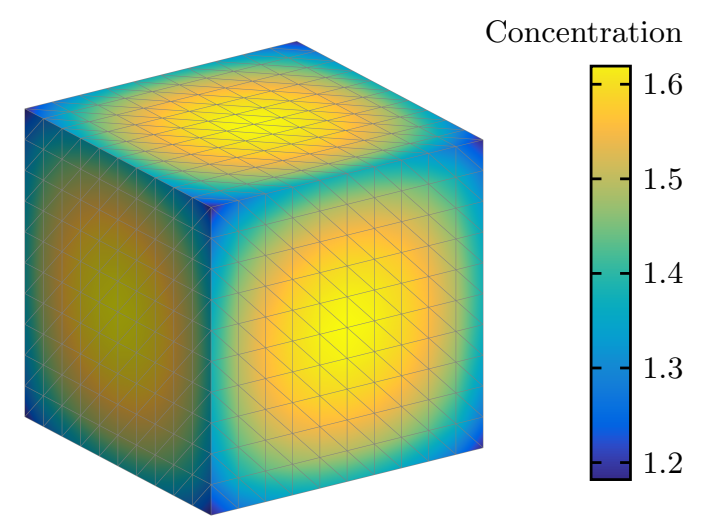

Fig. 7. Surface concentration of a cube with uniform activity $\mathcal{A}=1$ and sides of length $L=2$. The double layer constant $\lambda=1 / 2,3 / 7,7 / 8$ for the faces, edges, and corners respectively.

even with 200 non-zero coefficients the analytical slip velocity is only suitable for a qualitative comparison.

The code was validated for regular meshes with 1026 and 4098 linear nodes, for which the relative error in the calculated swimming velocity was $4.5 \%$ and $0.4 \%$ respectively. The concentration field and slip velocity are shown together in figure 6 for the refined mesh. The mean relative error in the surface concentration is $0.8 \%$. While the cusp in the slip velocity may not be fully captured by our model, this clearly does affect the calculation of swimming velocities. Away from the cusp, the mean error in the slip velocity is $0.7 \%$, though due to the slow convergence of the series a more accurate estimate is not possible.

\section{Discussion}

In this work, we have proposed a generic and versatile pipeline to solve phoretic motion problems in the classical continuum framework. The method relies on successive boundary element solutions of the Laplacian, using regularised sourcelets, and the Stokes flow equations using regularised stokelets [23]. The method fully exploits 


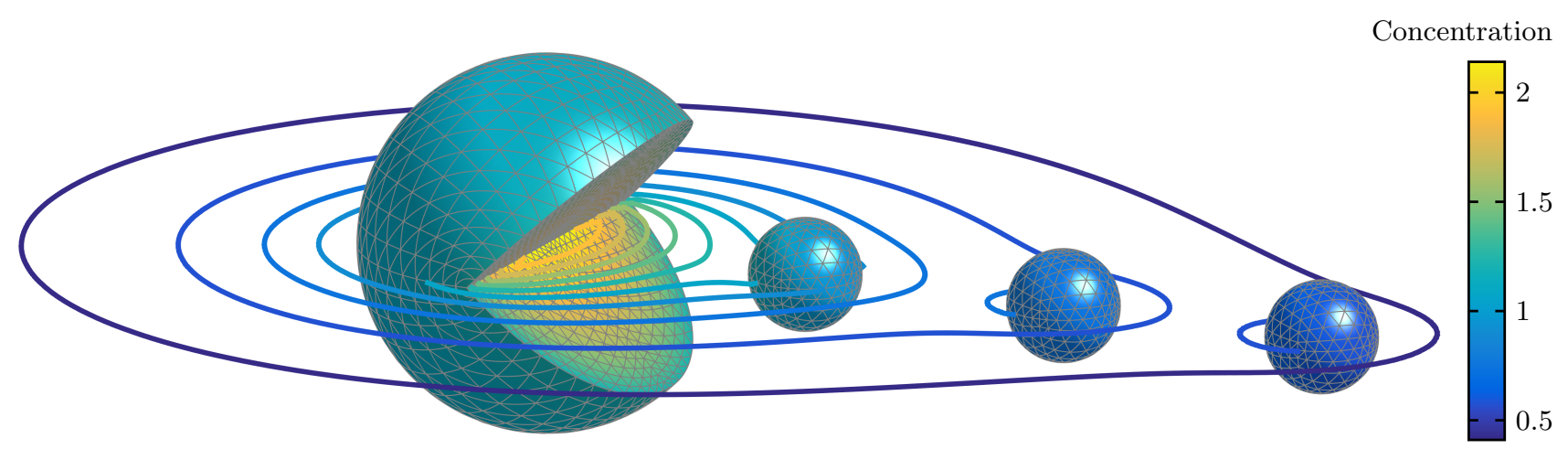

Fig. 8. Surface concentration and contours of the concentration field in the bulk for a non-trivial geometry with multiple particles: a phoretic pacman interacting with phoretic pacdots. Activity is uniform, $\mathcal{A}=1$ for all surfaces.

the advantages of boundary element methods for phoretic problems; the solute diffusion and Stokes flow problems are only coupled on the boundary. The method has been validated against analytical solutions for two separate phoretic systems, entailing multiple particles and discontinuous flux conditions. In contrast with simulation techniques requiring a computational mesh of the entire fluid domain, dynamic simulations of multiple moving particles require no re-meshing between time-steps, providing a computationally efficient approach. This method may prove particularly useful to address problems with complex geometries that do not readily admit an analytical solution; figure 7 shows the surface concentration of a cube with uniform activity. The approach could equally be used to examine L-shaped particles [38] without the slender body approximation. Furthermore, the method can be applied for multiple particle systems with non-trivial geometries; figure 8 shows the surface concentration and midplane concentration contours of a phoretic pacman of uniform activity, ready to exploit the concentration gradient generated by his mouth to eat the uniformly active pacdots.

\section{Acknowledgements}

TDMJ is supported by a Royal Commission for the Exhibition of 1851 Research Fellowship. SM acknowledges the support of the French Ministry of Defense (DGA). This work was funded in part by a European Union Marie Curie CIG Grant to EL.

\section{References}

1. E. Lauga and T. R. Powers. The hydrodynamics of swimming micro-organisms. Rep. Prog. Phys., 72:096601, 2009.

2. B. J. Nelson, I. K. Kaliakatsos, and J. J. Abbott. Microrobots for minimally invasive medicine. Ann. rev. biomed. eng., 12:55-85, 2010.

3. A. Ghosh and P. Fischer. Controlled propulsion of artificial magnetic nanostructured propellers. Nano Lett., 9:2243$2245,2009$.
4. F. A. Godnez, L. Koens, T. D. Montenegro-Johnson, R. Zenit, and E. Lauga. Complex fluids affect low-reynolds number locomotion in a kinematic-dependent manner. Exp. Fluids, 56(5), 2015.

5. S. J Ebbens and J. R Howse. In pursuit of propulsion at the nanoscale. Soft Matter, 6(4):726, 2010.

6. A. Walther and A.H.E. Müller. Janus particles. Soft Matter, 4(4):663-668, 2008.

7. J. L. Anderson. Colloid transport by interfacial forces. Ann. Rev. Fluid Mech., 21:61-99, 1989.

8. W F Paxton, K C Kistler, C C Olmeda, A Sen, S K St Angelo, Y Cao, T E Mallouk, P E Lammert, and V H Crespi. Catalytic Nanomotors: Autonomous Movement of Striped Nanorods. J. Am. Chem. Soc., 126(41):13424-13431, 2004.

9. J. R. Howse, R. A. L. Jones, A. J. Ryan, T. Gough, R. Vafabakhsh, and R. Golestanian. Self-Motile Colloidal Particles: From Directed Propulsion to Random Walk. Phys. Rev. Lett., 99(4):048102, 2007.

10. A. Brown and W. Poon. Ionic effects in self-propelled ptcoated janus swimmers. Soft Matter, 10:4016-4027, 2014.

11. I. Theurkauff, C. Cottin-Bizonne, J. Palacci, C. Ybert, and L. Bocquet. Dynamic clustering in active colloidal suspensions with chemical signaling. Phys. Rev. Lett., 108:268303, 2012.

12. R. Golestanian, T. B. Liverpool, and A. Ajdari. Designing phoretic micro-and nano-swimmers. New J. Phys., 9(5):126, 2007.

13. S. Shklyaev, J. F. Brady, and U. M. Cordova-Figueroa. Non-spherical osmotic motor: chemical sailing. J. Fluid Mech., 748:488-520, 2014.

14. S. Michelin and E. Lauga. Autophoretic locomotion from geometric asymmetry. Euro. Phys. J. E, 38(2):1-16, 2015.

15. S. Reigh and R. Kapral. Catalytic dimer nanomotors: continuum theory and microscopic dynamics. Soft matter, 11(16):3149-3158, 2015.

16. F. Sciortino, A. Giacometti, and G. Pastore. A numerical study of one-patch colloidal particles: from square-well to janus. Phys. Chem. Chem. Phys., 12(38):11869-11877, 2010.

17. P. K. Ghosh, V. R. Misko, F. Marchesoni, and F. Nori. Self-propelled janus particles in a ratchet: Numerical simulations. Physical review letters, 110(26):268301, 2013.

18. R. Soto and R. Golestanian. Self-assembly of catalytically active colloidal molecules: tailoring activity through surface chemistry. Phys. Rev. Lett., 112:068301, 2014. 
19. W. E. Uspal, M. N. Popescu, S. Dietrich, and M. Tasinkevych. Self-propulsion of a catalytically active particle near a planar wall: from reflection to sliding and hovering". Soft Matt., 11:434-438, 2015.

20. R. Singh, S. Ghose, and R. Adhikari. Many-body microhydrodynamics of colloidal particles with active boundary layers. J. Stat. Mech.: Theory and Exp., 2015(6):06017, 2015.

21. S. Michelin, T. D. Montenegro-Johnson, G. De Canio, N. Lobato-Dauzier, and E. Lauga. Geometric pumping in autophoretic channels. Soft matter, 2015.

22. R. Cortez. The method of regularized stokeslets. SIAM J. Sci. Comput., 23(4):1204-1225, 2001.

23. R. Cortez, L. Fauci, and A. Medovikov. The method of regularized Stokeslets in three dimensions: analysis, validation, and application to helical swimming. Phys. Fluids, 17(031504):1-14, 2005.

24. D. J. Smith. A boundary element regularized Stokeslet method applied to cilia- and flagella-driven flow. Proc. $R$. Soc. Lond. A, 465:3605-3626, 2009.

25. S. Gueron and N. Liron. Ciliary motion modeling and dynamic multicilia interactions. Biophys. J., 63:10451058, 1992.

26. J. Ainley, S. Durkin, R. Embid, P. Boindala, and R. Cortez. The method of images for regularized Stokeslets. J. Comput. Phys., 227(9):4600-4616, 2008.

27. T. Ishikawa, M.P. Simmonds, and T.J. Pedley. Hydrodynamic interaction of two swimming model microorganisms. J. Fluid Mech., 568:119-160, 2006.

28. http://uk.mathworks.com/matlabcentral/profile/ authors/5102158-thomas-montenegro-johnson.

29. P.O. Persson and G. Strang. A simple mesh generator in MATLAB. SIAM review, 46(2):329-345, 2004.

30. J.H.M. Frijns, S.L. De Snoo, and R. Schoonhoven. Improving the accuracy of the boundary element method by the use of second-order interpolation functions. Biomedical Engineering, IEEE Transactions on, 47(10):1336-1346, 2000.

31. M.A. Taylor, B.A. Wingate, and R.E. Vincent. An algorithm for computing fekete points in the triangle. SIAM Journal on Numerical Analysis, 38(5):1707-1720, 2000.

32. A.A. Smith, T.D. Johnson, D.J. Smith, and J.R. Blake. Symmetry breaking cilia-driven flow in the zebrafish embryo. J. Fluid Mech., 705:26-45, 2012.

33. https://people.sc.fsu.edu/ jburkardt.

34. C. Pozrikidis. A practical guide to boundary element methods with the software library BEMLIB. CRC Press, 2002.

35. H. Guo, J. Nawroth, Y. Ding, and E. Kanso. Cilia beating patterns are not hydrodynamically optimal. Phys. Fluids, 26(9):091901, 2014.

36. S. Michelin and E. Lauga. Phoretic self-propulsion at finite péclet numbers. J. Fluid Mech., 747:572-604, 2014.

37. S. Michelin, E. Lauga, and D. Bartolo. Spontaneous autophoretic motion of isotropic particles. Phys. Fluids, 25:061701, 2013.

38. B. Ten Hagen, F. Kümmel, R. Wittkowski, D. Takagi, H. Löwen, and C. Bechinger. Gravitaxis of asymmetric self-propelled colloidal particles. Nature communications, 5, 2014. 